\title{
Green Synthesis and Characterization of Silver Nanoparticles using Leaf Extract of Tridax procumbens
}

\author{
JYOTI V.VASTRAD* and GIRIDHAR GOUDAR
}

\begin{abstract}
DBT Project, All India Co-ordinated Research Project-Home Science (Clothing \& Textiles), Research Complex, University of Agricultural Sciences, Dharwad, Karnataka, India

${ }^{*}$ Corresponding author E-mail: jyotivastrad@gmail.com
\end{abstract}

http://dx.doi.org/10.13005/ojc/320327

(Received: March 27, 2016; Accepted: May 03, 2016)

\begin{abstract}
An ecofriendly approach for green synthesis of nanoparticles using natural plant extracts is gaining a notable importance now a days. In the present study, Tridax procumbens leaf has been used to produce the silver nanoparticles (AgNps) from two solvent systems (distilled water and $50 \%$ alcohol). Biosynthesis of AgNps from the leaf extracts was carried out and the characterization of the synthesized AgNps was done using UV-Visible spectroscopy, Particle Size Analysis and Scanning Electron Microscope (SEM). Both the extracts exhibited significant results for the biosynthesis of AgNps by using silver nitrate as a reducing agent, the synthesis of AgNps was assertained by colour change from yellowish green to dark brown. The UV-Visible spectroscopy revealed the absorption maxima at $230 \mathrm{~nm}$ and $235 \mathrm{~nm}$ for distilled water and $50 \%$ alcohol AgNps respectively. The nanoparticle sizes were in the range from $20-154 \mathrm{~nm}$ which was ascertained from Particle Size Analysis and Scanning Electron Microscope (SEM). The use of nanotechnology in the textile industry has increased rapidly due to its unique and valuable properties. Also, there is an considerable potential for profitable applications of nanotechnology in cotton and other textile industries.
\end{abstract}

Keywords: Tridax procumbens, nanoparticles, UV-Visible spectroscopy, SEM.

\section{INTRODUCTION}

Nanotechnology has emerged as the interdisciplinary science conjugating the basic concept of engineering with the application of biology, chemistry, physics and medical science'. Nanotechnology is the science of the small, the very small. It is the use and manipulation of matter at a tiny scale. At this size, atoms and molecules work differently, and provide a variety of surprising and interesting uses ${ }^{2}$. Nanoparticles are being viewed as fundamental building blocks of Nanotechnology ${ }^{3}$. Nanotechnology represents the design, production and application of materials at atomic, molecular and macromolecular scales, in order to produce new nanosized materials ${ }^{4}$. Nanotechnology has 
diversified into various branches, encompassing new approaches based upon molecular self assembly for developing new materials of less than $100 \mathrm{~nm}$ range ${ }^{5}$. Nanotechnology has real commercial potential for the textile industry. This is mainly due to the fact that conventional methods used to impart different properties to fabrics often do not lead to permanent effects, and will lose their functions after laundering or wearing ${ }^{6}$.

Metal nanoparticles have attained a great importance due to their unique feature such as catalytic, magnetic, optical and electrical properties ${ }^{7,8}$. Silver nanoparticles (AgNPs) have attracted considerable interest in biological studies because of their ease of preparation, good biocompatibility, and relatively large surface area. The synthesis of nanoparticles of different chemical compositions, sizes, and controlled monodispersity is an important area of research in nanotechnology ${ }^{9}$.

Plants are known to possess various therapeutic compounds which are being exploited since ancient time as a traditional medicine. Recent reports of plants towards production of nanoparticles is said to have advantages such as easily available, safe to handle and broad range of biomolecules such as alkaloids, terpenoids, phenols, flavanoids, tannins, quinines etc. are known to mediate synthesis of nanoparticles ${ }^{10}$.

Tridax procumbens Linn. belongs to the family Compositae. It is commonly known as 'Common button' or 'Coat button' and it is a weed found throughout India. A hispid, procumbent herb with woody base sometime rooting at the node, upto $60 \mathrm{~cm}$ high. Leaves are ovate-lanceolate 2 to $7 \mathrm{~cm}$ and lamina pinnatisect, sometimes three lobed ${ }^{11}$. The leaf juice possesses antiseptic, insecticidal and parasiticidal properties and is used also to check haemorrhage from cuts, bruises and wounds ${ }^{12}$. The leaf has been proved to have wound healing property ${ }^{13}$.

Biosynthetic and physicochemical approaches ${ }^{14}$ have been extensively used to prepare silver nanoparticles with the aim of improving the conventional methods. A variety of chemical approaches have also been utilized to produce silver nanoparticles with different size distribution and different shapes ${ }^{15,16}$. Silver nanoparticles have received considerable attention as antimicrobial agents and have been shown to be an effective antimicrobial agent ${ }^{17}$.

Present study is the outcome of the research work conducted to synthesize and characterize AgNps using T.procumbens plant leaf extracts.

\section{MATERIAL AND METHODS}

Fresh leaves of T.procumbens plant were selected for the study that were collected from the campus of University of Agricultural Sciences, Dharwad, Karnataka.

\section{Chemicals}

Analytical reagent (AR) grade silver nitrate and ethyl alcohol was purchased from Thomas Baker, Mumbai.

\section{Extraction of bioactive compounds}

The leaves of T.procumbens were cleaned with distilled water and shade dried for 2 hours to remove moisture from the surface. Two grams each of fresh leaf was weighed, chopped into fine pieces and ground in a laboratory mortar and pestle. Finely ground leaf were mixed in the solvents; $50 \%$ ethyl alcohol ( $\mathrm{v} / \mathrm{v})$ and distilled water, $25 \mathrm{ml}$ of each solvent. Centrifuged at $4^{\circ} \mathrm{C}$ for 10 minutes at 10,000 rpm (Remi Elektrotechnik, C24 Plus, Mumbai) and supernatant was collected. Residue was re-extracted with fresh $25 \mathrm{ml}$ of the respective solvent and the process was repeated. The supernatant obtained was pooled and stored under refrigeration at $8^{\circ} \mathrm{C}$ until further analysis.

\section{Biosynthesis of silver nanoparticles (AgNps)}

To $100 \mathrm{ml}$ of $1 \mathrm{mM} \mathrm{AgNO}_{3}$ solution, $5 \mathrm{ml}$ of leaf extract of each solvent (50\% ethyl alcohol and distilled water) was added separately to two conical flasks, stirred at $200 \mathrm{rpm}$ using magnetic stirrer for $3 \mathrm{hrs}$ and kept under dark at room temperature. The colour change was observed from light yellowish green to brown colour within 10 minutes. The incubation time was continued for a week and the change in colour of the solution was recorded. 


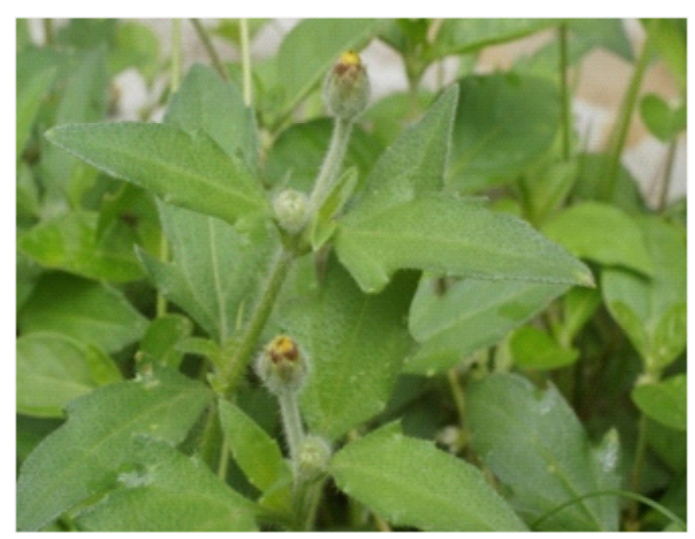

Fig. 1: Tridax procumbens leaf
Characterization of Silver nanoparticles (AgNps) UV-Visible Spectroscopy

Spectroscopy analysis of biosynthesized silver nanoparticles was carried out using UV Visible Spectrophotometer (Biomate 3S, Thermo Scientific, USA) as a function of time needed for bioreduction at room temperature. The gradual change in the colour of samples from light yellow to dark brown colour was observed and the bioreduction of $\mathrm{Ag}+$ in the solvent extracts was monitored by periodic evaluation of the suspension $(2 \mathrm{ml})$ after each $24 \mathrm{~h}$ of incubation under dark condition till one week, the aliquots were subsequently measured for the UV-Visible spectra by scanning in the region from $200-700 \mathrm{~nm}$.
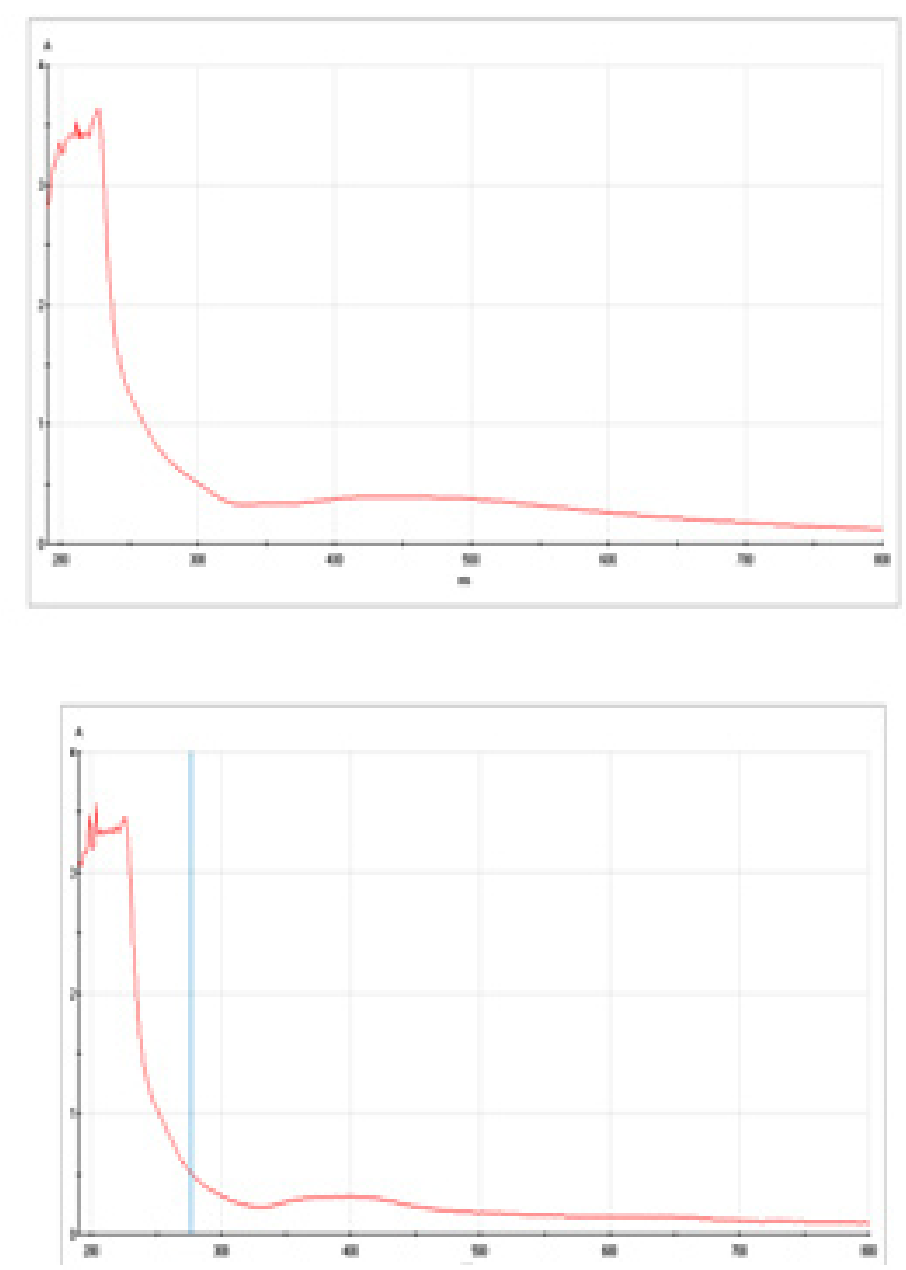

Fig. 2: UV-Visible absorbance curve of T.procumbens silver nanoparticle (a) Distilled water extract (b) $50 \%$ ethyl alcohol extract 


\section{Particle size analysis}

There are different techniques for the measurement of particle size and its distribution (PSD) such as sieve analysis, optical counting methods, electro resistance counting methods, sedimentation techniques, laser diffraction methods, dynamic light scattering (DLS) method and acoustic spectroscopy. Among them light scattering is used for
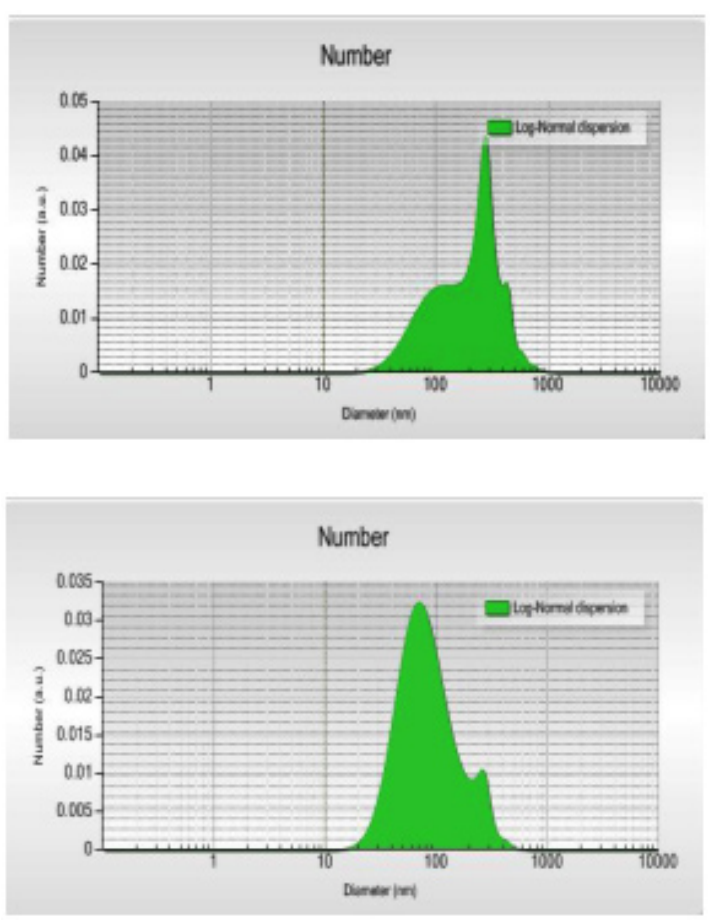

obtaining size distribution of nanoparticles. The size and size distribution have been studied for the AgNps prepared from the plant extracts by DLS technique.

\section{Scanning Electron Microscope (SEM)}

SEM analysis was undertaken to know the size and shape of the silver nanoparticles biosynthesized using the plant leaf extracts of
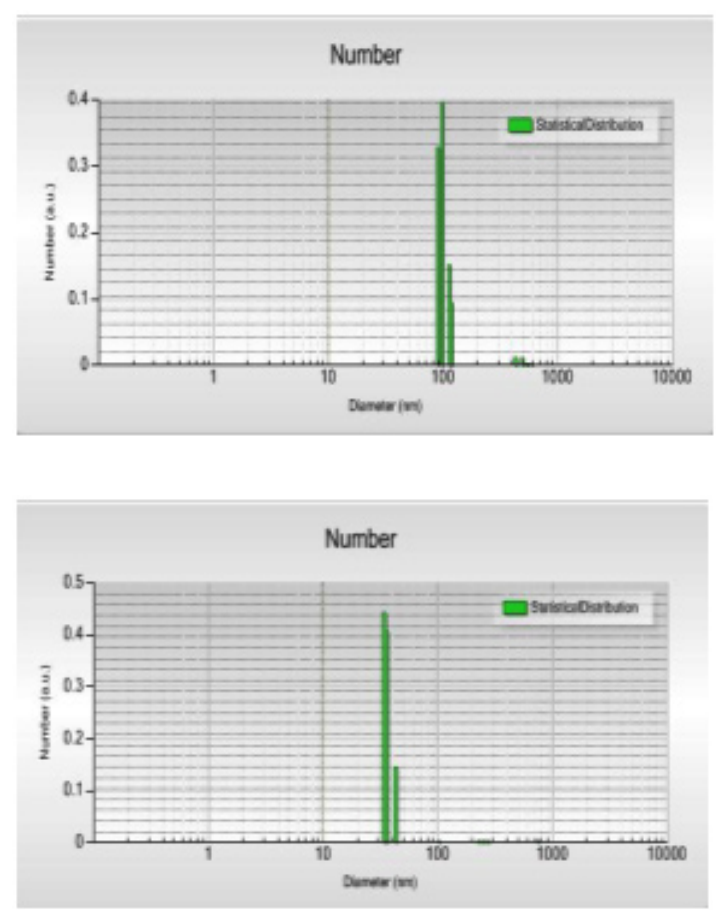

Fig. 3: Particle size analysis of T.procumbens silver nanoparticle (a \& b) $50 \%$ ethyl alcohol extract (c \& d) Distilled water extract $\mathrm{H} 2 \mathrm{O}$ extract
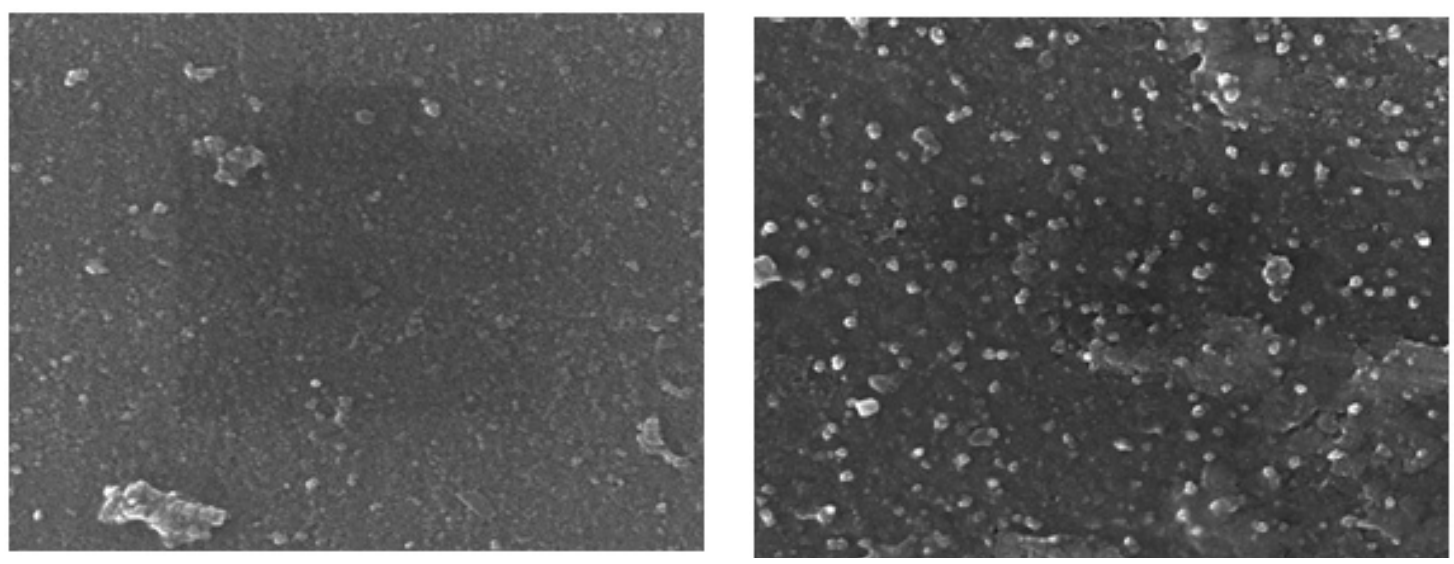

Fig. 4: SEM analysis of T.procumbens silver nanoparticle

(a) Distilled water extract (b) $50 \%$ ethyl alcohol extract 
T. procumbens. The analysis was done using $\mathrm{FEI}$ Quanta 200 SEM machine. Thin films of the sample were prepared on a carbon coated copper grid by just dropping a very small amount of the sample on the grid, extra solution was removed using a blotting paper. Then the film on the SEM grid was allowed to dry and the images of nanoparticles were taken.

\section{RESULTS}

\section{Characterization of Silver nanoparticles (AgNps) UV- Visible Spectroscopy}

UV-Visible spectroscopy analysis of AgNps from aqueous and $50 \%$ ethyl alcohol extracts of T. procumbens leaves were evaluated. Reduction of the silver ion to silver nanoparticles during exposure to the plant leaf extracts was followed by color change of the solutions. Figure 2 shows the UV-Visible absorption spectra recorded for the silver nanoparticle solutions after $24 \mathrm{hrs}$ of reaction time.

The strong absorption maxima was obtained at $230 \mathrm{~nm}$ and a small shoulder peak at $400 \mathrm{~nm}$ was obtained for the AgNp solution of aqueous extract (Fig. 2a). Whereas for the AgNPs from $50 \%$ ethyl alcohol extract revealed the strong absorption maximum at $235 \mathrm{~nm}$ and a specific shoulder peak at 390nm (Fig. 2b). It is well known that silver nanoparticles exhibit yellowish brown color in aqueous solution due to excitation of surface plasmon vibrations in silver nanoparticles $(18,19)$.

\section{Particle size analysis}

Particle size analysis by dynamic light scattering (DLS) method revealed the size distribution of the synthesized AgNps, which were in the range between $97-419 \mathrm{~nm}$ for the aqueous leaf extract of T. procumbens (Fig. 3a,b), whereas the particle sizes of the AgNps from $50 \%$ ethyl alcohol leaf extract were in the range from 20-75nm (Fig. 3c,d).

\section{Scanning Electron Microscope (SEM)}

SEM analysis of the silver nitrate solution (Control) and reduced form of silver nitrate solution were clearly distinguishable owing to their size difference. The SEM image shows the AgNps synthesized from $T$. procumbens extracts (aqueous $\& 50 \%$ ethyl alcohol) which is further confirmation of the presence of AgNps. The shape of the AgNps in T. procumbens aqueous extract was spherical and cubic; the sizes of AgNps were 97.32 and 154.64nm as confirmed by SEM image (Fig. 4a). Also, varying sizes of AgNps were observed in the T. procumbens $50 \%$ ethyl alcohol extract, the sizes of AgNps were $57.11,68.47$ and $86.23 \mathrm{~nm}$ which were comparatively smaller when compared with AgNPs of aqueous extract.

\section{DISCUSSION}

The results for AgNps synthesized from aqueous and $50 \%$ ethyl alcohol showed the absorbance in the UV-Visible region between 220-240nm. In a similar study on AgNps using pennyroyal leaf extract, the UV-Vis absorption spectra was recorded for AgNP solution and the maximum absorption was at $490 \mathrm{~nm}^{20}$ and a study on AgNPs of Solanum nigrum-mediated silver nanoparticles revealed the absorbance spectrum of AgNPs formed at various times $(0,2,4,6 \mathrm{~h})$ and the maximum absorption peak was observed in the range of 370-420 nm and the absorption spectrum of spherical AgNPs was at $420 \mathrm{~nm}^{21}$. The particle size and SEM analysis revealed that the sizes of the AgNps prepared were in the range between 20-154nm. The AgNps derived from T. procumbens leaves may have the antimicrobial property which needs to be studied in detail against specific microorganisms. The AgNps from natural source can be synthesized which can have several beneficial effects for specific application.

\section{CONCLUSION}

In the present study, a simple and economic approach has been attempted to obtain a green ecofriendly synthesis of silver nanoparticles which was obtained from bio-reduction of T. procumbens leaf extracts with $\mathrm{AgNO}_{3}$ solution. Silver nanoparticles synthesized by the green chemistry approach reported in the present study may have potent applications in functional textiles. Synthesized AgNPs from the plant extracts are characterized specifically using UV-Visible spectroscopy, Particle Size Analyzer and SEM, whereas protocol to produce uniform sized nanoparticles has to be standardized for specific applications. 


\section{REFERENCES}

1. Prabhu, S.; Poulose, E.K. Int. Nano Lett. 2012, 11. 2(32), 1-10.

2. Sovan, L. P.; Utpal, J.; P. K. Manna; G. P. Mohanta; R. Manavalan. J App. Pharm. Sci. 2011, 01(06), 228-234.

3. Thirumurugan, A.; Neethu, A. T.; Hema, P. K.; Prakash, P. International Journal of Nanomaterials and Biostructures. 2011, 1(2), 22-24.

4. Hahens, W. I.; Oomen, A. G.; deJong, W. H.; Cassee, F. R. Regulatory Toxicology and Pharmacology. 2007, 49, 217-229.

5. Karunakar, R. K.; Manisha, R. D.; Prashanthi, Y.; Ramachander, M.; PratapRudra, M. P. Int. J. Res. Pharm. Sci. 2013, 4(4), 504-511.

6. Laleh, M.; Abo, S. R.; Nahid, A. G. Orient. J. Chem. 2015, 31(1), 257-262.

7. Garima, S.; Bhavesh, R.; Kasariya, K.; Ranjan, A.S.; Singh, R.P. J. Nanopart. Res. 2011, 13, 2981-2988.

8. Vidhya, C.; Shilpa, H.; Chandraprabha, M.N.; Antonyraj, M.A.L.; Indu, V.G.; Aayushi, J.; Bansal, K. IJCET. 2013, 118-120.

9. Hamid, R. G.; Saeid, S. Orient. J. Chem. 2015, 31(1), 341-344.

10. Vastrad, J. V.; Goudar, G. Res. J. Chem. Environ. 2015, 19(11), 1-7.
11. Ganju, K.; Pathak, A.K. J. Pharmacogn Phytochem. 2013, 1(5), 42-46.

12. Saxena, V.K.; Albert, S. J. Chem. Sci. 2005, 117(3), 263-266.

13. Raina, R.; Prawez, S.; P. K. Verma.; N. K. Pankaj. VetScan. 2008, 3(1), 221-224.

14. Chaudhari, P. R.; Masurkar, S. A.; Shidore, V. B.; Kamble, S. P. J. App. Pharmaceutical Sci. 2012, 02, 25-29.

15. Prabhu, N.; Divya, T.R.; Yamuna, G. Digest. J. Nanomater. Biostruct., 2010, 5, 185-189.

16. Khan, Z.; Al-Thabaiti, S.A.; Obaid, A.Y.; AlYoubi, A.O. Colloid. Surf. B: Biointerfaces, 2011, 82, 513-517.

17. Galdiero, S.; Falanga, A.; Vitiello, M.; Cantisani, M.; Marra, V.; Galdiero, M. Molecules. 2011, 16, 8894-8918.

18. Shankar, S.S.; Rai, A.; Ahmad, A.; Sastry, M. J. Colloid Interf. Sci. 2004, 275(2), 496-502.

19. Ankamwar, B.; Chaudhary, M.; Sastry, M. Synth. React. Inorg. Metal Org. Nano Metal Chem. 2005, 35(1), 19-26.

20. Sajjad, S.; Arezoo, E. A.; Siamak, B. J. Nanostruct. Chem. 2016, 6, 25-27.

21. Arumugam, S.; Adithan, A.; Chinnapan, S.; Kandasamy, S.; Palanisamy, S.; Muthusamy, G.; Koildhasan, M,; Thangaswamy, S. J. Nanostruct. Chem. 2016, 6, 41-48. 